\title{
Status of argasid (soft) ticks (acari: parasitiformes: argasidae) in relation to transmission of human pathogens
}

\begin{abstract}
Ticks transmit a greater variety of infectious agents than any other arthropod group, in fact, these are second only to mosquitoes as carriers of human pathogens. This article concerns to the different ticks as vectors of parasites and their control methods having a major focus on vaccines against pathogens. Typically, argasids do not possess a dorsal shield or scutum, their capitulum is less prominent and ventrally instead anteriorly located, coxae are unarmed (without spurs), and spiracular plates small. A number of genera and species of ticks in the families Argasidae (soft ticks) are of public health importance. Certain species of argasid ticks of the genera Argas, Ornithodoros, Carios and Otobius are important in the transmission of many human's pathogens. Moreover, argasids have multi-host life cycles and two or more nymphal stages each requiring a blood meal from a host. Unlike the ixodid (hard) ticks, which stay attached to their hosts for up to several days while feeding, most argasids are adapted to feed rapidly (for about an hour) and then dropping off the host. They transmit a variety of pathogens of medical and veterinary interest, including viruses, bacteria, rickettsiae, helminthes, and protozoans, all of which are able to cause damage to livestock production and human health. Control of tick infestations has been mainly based on the use of acaricides, which is a control measure with serious drawbacks, due to liable for the contamination of products, as acaricide-resistant pests and an environmental contaminant. It is important to evaluate clinical findings and history of exposure carefully. Awareness is the only defense in case of no vaccines availability for pathogens and healthcare workers need to be more aware (correct tick removal, recognition of potential infection and prompt treatment). To avoid long-term and irreversible effects of infection, early recognition of infection, timely initiation of treatment, and careful monitoring during and post treatment are recommended. Research on alternatives to the use of acaricides is strongly represented by tick's infection vaccines by considering a more cost-effective and environmentally safe strategy.
\end{abstract}

Keywords: ticks, argasidae, tick-borne diseases, control methods, human pathogens
Volume 4 Issue 4 - 2017

\author{
Muhammad Sarwar \\ Department of Entomology, Nuclear Institute for Food \& \\ Agriculture (NIFA), Pakistan
}

Correspondence: Muhammad Sarwar, Department of Entomology, Nuclear Institute for Food \& Agriculture (NIFA), Pakistan, Email drmsarwar64@yahoo.com

Received: December II, 2016 | Published: September 21, 2017

\section{Introduction}

Ticks are distributed worldwide and have an enormous medical and veterinary importance owing to the direct damage they cause to their hosts, and especially because these are vectors of a large variety of human and animal pathogens. In fact, ticks are second to mosquitoes as vectors of human pathogens and the most important vectors of pathogens affecting cattle worldwide. ${ }^{1-3}$ Tick species can be grouped in two main families, the Argasidae (soft ticks), and the Ixodidae (hard ticks). A third tick family, Nuttalliellidae, only has one species, Nuttalliella namaqua (represented by a monotypic species restricted to South Africa). These three families share common basic properties that are modified distinctively inside each family according to their particular behavior patterns and life-style. ${ }^{4}$ They transmit a variety of pathogens of medical and veterinary interest, including viruses, bacteria, rickettsiae, helminthes, and protozoans, all of which are able to cause damage to livestock production and human health. The global threat of tick-borne diseases is increasing, with new pathogens identified continuously. In humans, tick infestations typically involve few specimens and the greatest risk for people bitten by a tick lies in infection due to tick-borne pathogens. Such pathogens are diverse and mainly include viruses, bacteria, and protozoa. ${ }^{5}$ The most commonly observed human tick-borne diseases are reportedly Lyme disease, tick-borne encephalitis, Crimean-Congo hemorrhagic fever, Q fever, tularemia, and North-Asia tick-borne spotted fever. Epidemiologically important tick-borne diseases, such as human granulocytic anaplasmosis and severe fever with thrombocytopenia syndrome, have also emerged in recent years. The characterization of a new bunyavirus (associated with fever, thrombocytopenia and leukopenia syndrome) has prompted greater attention to ticks and tick-borne diseases. ${ }^{6}$

Tick ecto-parasites have direct impact on the vertebrate hosts. Direct injuries to humans can be very serious, especially in tropical climates, and are mainly observed in infestations with ixodid ticks, but also in infestations with some argasid ticks as Ornithodoros lahorensis and $O$. savignyi. ${ }^{7}$ The most frequent of these direct forms of damage include; (i) tissue destruction caused by the tick mouth parts and by the local inflammatory reaction of host to tick saliva; (ii) loss of blood, toxicoses and immunosuppression. Apart from these direct effects, the most important feature of ticks is that they are vectors as well as reservoirs of multiple pathogens. ${ }^{8}$

The Argasidae is the family of soft ticks and includes some 193 species, so, it is one of the two big families of ticks. They lack the hard scutum present in the hard ticks (Ixodidae), the capitulum (mouthpartsbearing structure) is located on the underside of the animal's body and is not readily visible, while in the Ixodidae the capitulum projects forward from the body. Although the family contains many species and the composition of the genera is less certain, so, more study is needed before the genera can become stable. The currently accepted genera are Antricola, Argas, Nothoaspis, Ornithodoros and Otobius. ${ }^{9}$ 
Argasid ticks differ from ixodids in a range of morphological and biological characteristics. The Argasidae are characterized by a tough leathery integument in all but the larval stage. Eyes when present are on the lateral surface of the body. The capitulum is recessed ventrally near the anterior end and is not visible dorsally (except in larvae). Nymphal and adult argasids also bear a pair of tiny pores, coxal pores, representing the openings of the coxal glands, located between the paired coxae of legs I and II. Excess fluid filtered from the blood meals they take from host is excreted via these pores. The spiracles or stigmata occur in the supracoxal folds between the coxae of legs III and IV. ${ }^{4}$ Some soft tick species exhibit extremely rigid host specificity. However, it has been suggested that most soft ticks show indiscriminate host feeding, and such apparent variation in host preference probably reflects microhabitat preference and host availability within the microhabitat. Most argasids are fast feeders, ingesting a relatively small amount of blood per meal and adult specimens can feed and reproduce repeatedly. Argasids are very resistant to starvation and can survive for several years without feeding. ${ }^{10}$ This, and their diapause periods, afford them with great flexibility in their developmental cycles. ${ }^{11}$ Species of the following genera have been identified, Argas (seven species), Carios (four species) and Ornithodoros (two species) in the family Argasidae. In the Argasidae, adults become sexually active after emergence from the last nymphal molt and they do not require a blood meal to initiate gametogenesis. Mating occurs before, as well as after blood feeding, but rarely if ever on the host itself. However, the gonotrophic cycle is completely dependent upon the blood meal (except in those rare instances in which mated females oviposit autogenously i.e., without feeding). In Argasidae, there are more than four developmental stages in the life cycle; egg, larva, several nymphal stages and adult. The typical argasid life cycle is a multi-host life cycle with multiple parasitic phases and repeated gonotrophic cycles. However, there are some noteworthy exceptions e.g., Ornithodoros lahorensis, which are 2-host ticks or Otobius megnini and O. lagophilus, which are 1-host ticks. Following embryogenesis and hatching the hungry larvae attack vertebrate hosts inhabiting the nest, burrow, or other niche environment. Following host contact, larvae attack it and feed rapidly (15-30minutes) and drop to molt in the sand, duff or cracks and crevices of the natural habitat. Fed larvae molt to first stage nymphs resembling miniature adults in body characteristics, especially the leathery, mammillae body cuticle, but lacking the genital pore and any evidence of sexual dimorphism. In some argasid species, larvae do not feed, but molt directly, while in others, larvae feed slowly and molt twice. Following engorgement, fed larvae detach from host, drop off and ecdyse by molting into the first nymphal stage. Hungry nymphs again attack to hosts that enter the niche thus repeating the cycle. This cycle of host contact, rapid feeding, engorgement, detachment and ecdysis in the niche occurs several times indicating the repeat of these processes. Diapause is a major factor regulating the time of development of many of the argasid species, which must survive in empty burrows or nests for periods of many months until their hosts return or new hosts arrive. ${ }^{12-14}$

Argasids tend to be endophilic/ nidicolous parasites that colonize the nests and burrows of their hosts and feed when the host arrives. Almost all argasid ticks have a multi-host feeding pattern that feed on mammals and birds (but excluding bats). An important factor enabling the nymphs to feed rapidly is their ability to eliminate excess blood meal water in the form of coxal fluid, which is a clear, colorless liquid excreted from the coxal glands during or shortly after feeding. A nutritional factor, especially blood volume taken in previous stages, is believed to be an important indicator of the number of nymphal stages. Moreover, males usually emerge sooner than females i.e., males require 1 or 2 fewer nymphal stages than do females. Nymphs have from two to eight separate instars before the ticks mature to adults. The exact number of instars varies according to the species and its future sex when adult. It is also influenced by the individual's state of nutrition. The precise number of nymphal stages as many as 8 nymphal instars has been recorded in some species. Following emergence, adults may first mate in the niche or attack hosts. Following host contact, adults feed rapidly, engorge and drop off in sheltered locality. Mated females oviposit small egg clutches, whereupon they return to attack hosts and feed again. Adults seek hosts, feed and engorge several times and fed mated females oviposit after each blood meal. Argasid ticks feed rapidly (except in larvae of certain Argas and Ornithodoros species), and the females feed and oviposit frequently (i.e., multiple gonotrophic cycles). The mated females deposit small egg masses ( $<500$ eggs per cycle). In the Argasidae, the passage of so many nymphal stages contributes to a much longer life cycle than in the Ixodidae. In addition, many argasid ticks can resist long periods of starvation during their development, so that the life cycle can be extended for many years. ${ }^{15,16}$

\section{Role of argasid as vectors of tick-borne pathogens in human}

Ticks can be carrier of any pathogens, which they transmit from host to host during blood sucking and cause a large variety of diseases. When a tick feeds it anchors its mouthparts deep within the skin and injects saliva. Excess water extracted from the ingested blood is voided back into the host through the salivary glands. Tick saliva contains bio-chemicals which numbs the bite area, keeps the blood flowing, prevents inflammation and dissolves tissue. If the tick is removed incorrectly (freezing, burning, squashing, scratching an attached tick, or applying solutions) this can cause it to regurgitate or spill saliva and gut contents, which may contain infective agents. The longer the tick remains attached, more saliva is pumped into the host to keep the blood flowing, numb the bite area and prevent inflammation. Hence, ticks especially the nymph and adult stages, are efficient transmitters of pathogens, namely viruses, bacteria and protozoa. ${ }^{17}$ Although the overall anatomy of the various species of argasids is similar, their functional approaches for feeding, reproduction, host finding and preference, host behavior, relationship to their environment, and disease association vary among species. ${ }^{18}$ There are 13 species of argasid ticks, belonging to three genera: Argas (seven species), Carios (four species), and Ornithodoros (two species) in China. The majority of these are nidicolous, usually residing in the burrows, caves, or nests of their hosts. Among all the argasids found, four Argas species, two Carios species and two Ornithodoros species are competent to transmit or cause human diseases. ${ }^{19}$ Among these eight tick species, four (A.japonicas, A. persicus, O. tartakovskyi and O. tholozani) have been confirmed as causing host illnesses. A case of human dermatitis has been recorded after a bite by $A$. japonicas, but no pathogen is identified from this tick species. The tick $A$. persicus mainly infests poultry and carries the most diverse array of pathogens in the family Agarsidae, including Borrelia anserine, Kyasanur forest disease virus and Wolbachia persica n. sp. However, only B. anserine is known to cause avian spirochetosis, which has been confirmed. Lake Clarendon virus is isolated from A. robertsi, Quaranfil virus and Gissar virus are identified in A. vulgaris, and Issyk-Kul virus has been identified in $C$. vespertilionis. The symptoms or diseases caused by these viruses are still unclear and the vector roles of these ticks remain unknown. The Carios capensis can be coinfected by pathogen DNA from Borrelia, Coxiella and Rickettsia, as well as West Nile virus, although no pathogens have been reported in this tick species collected. The Ornithodoros tartakovskyi and O. tholozani both 
cause tick-borne relapsing fever, but carry different pathogens, $B$. latyshevyi and B. persica, respectively. ${ }^{20}$ Harbour Argas reflexus (soft tick), can transmit Borreliosis to people and pets. Principal tick vector Argas hermanni transmits Royal Farm virus, while tick-borne relapsing fever is transmitted to humans through the bite of infected soft ticks. ${ }^{21}$ Soft ticks collected directly from wild and domestic birds and their nests have been tested for the presence of West Nile virus. The cattle egret argas, Argas arboreus, has been collected from the nests of seven cattle egret colonies. Out of 1,000 A. arboreus pools examined, 16 proved positive for virus based on RT-PCR technique from four nesting colonies of birds. Out of 37 cattle egret squabs examined, $37.8 \%$ has serum-neutralizing antibodies to virus. ${ }^{22}$ It is determined that ticks cause great economic losses in the world and adversely affect health in several ways and parasitize a wide range of vertebrate hosts, and transmit a wider variety of pathogenic agents than any other group of arthropods.

\section{Diagnosis of tick}

Diagnosis is made on physical examination of host, when attached ticks are recognized and recovered. Body of tick is oval or pearshaped, rounded anteriorly, mouth parts not visible from above (except in larva), but more easily seen from the ventral view. For submission to a diagnostic laboratory, ticks should be secured in specimen tubes with or without alcohol.

\section{Prevention and control of soft ticks}

Current strategies to control tick-borne diseases are largely based on measures to avoid exposure and to control vector species. In the area of tick control, much has been achieved, but much more remains to be done. The availability of vaccine is very small, but the ability to induce an effective, sustained immunological response is crucial, however, it needs further improvement. Problems of acaricide resistance, chemical residues in food and the environment, make the current situation unsatisfactory and require the development of absolute control through effective tick's infection vaccine. ${ }^{23}$ Control of West Nile virus can only be effective if the vectors and reservoirs of the virus are identified and controlled. Although mosquitoes are the primary vectors, Nile virus has repeatedly been isolated from ticks. Therefore, tick-borne transmission studies have been performed with an ixodid (Ixodes ricinus) and an argasid tick species (Ornithodoros moubata). Both species became infected after feeding upon viremic hosts, but, I. ricinus tick is unable to maintain the virus. In contrast, $O$. moubata tick remained infected for at least 132days, and the infection maintained through molting and a second blood meal. Infected $O$. moubata ticks transmitted the virus to rodent hosts, even though at a low level. Moreover, the virus is non-systemically transmitted between infected and uninfected $O$. moubata ticks co-fed upon uninfected hosts. Although ticks are unlikely to play a major role in Nile virus transmission, findings suggest that some species have the potential to act as reservoirs for the virus. ${ }^{24,25}$

\section{Surveillance of soft ticks}

Direct methods for tick surveillance are based on the capture and identification of specimens, either from the vegetation (dragging method) or from animal hosts in the area sampled. While these procedures are useful for the surveillance of ixodid ticks owing to their exophilous lifestyle and long feeding times, they may not work with argasid ticks because they are endophilous/nidicolous and fast feeders. This means that vegetation dragging and the removal from animals are inefficient as direct methods for argasid surveillance; instead it is necessary to explore all possible tick refuges in the area sampled before such an area can be considered tick-free. Evidently, these drawbacks have encouraged the development of serological tests (ELISA) as indirect methods for tick surveillance, especially for argasid ticks. Serological methods are based on the detection of specific antibodies against tick salivary proteins in serum samples taken from animal hosts or humans living in the area under study. ${ }^{26}$

\section{Chemical elimination of ticks}

Chemical elimination of ticks includes insecticide sprays and insecticide dusts. So far, the use of acaricides (pesticides that kill members of the arachnid subclass Acari, which also includes ticks) has been a major component of integrated tick control methods. The animal health authorities are treating cattle with a variety of chemical agents, mainly mixtures of querosene, sulphur and lard, in an effort to control ticks. Cyfluthrin, Lambda cyhalothrin, Ivermectin, Dicofol, Bifenthrin, Onslaught, Deltamethrin and Permethrin Pro are concentrates that can be used for outdoor and indoor tick's elimination. In areas where ticks are an on-going problem, spray shrubs, tree trunks, fences, ground covers and lawns every 3 months or so to keep pressure on the tick populations with a hose-end sprayer. Treat indoors with same concentrates using a pump type sprayer and repeat spray or dust applications in 7-0days as needed. ${ }^{27}$

\section{Safe remove of a tick}

Using tweezers, grasp the tick as close to skin as possible and gently pull it upward. Do not twist or jerk it, as it is wanted to keep the tick mouthparts and body intact. Also, do not grasp the tick around the body with tweezers or fingers. Likewise, it is also not wanted to squeeze the blood and other fluids from the engorged tick into the site of the bite. After removing the tick, disinfect the site of the bite. Intended for testing of ticks for disease, they must be kept alive. At a minimum, save the tick in a container with rubbing alcohol. Record the date and location from where are picked up the ticks whenever these are found attached. Take care, tick should be taken out slowly and with care as to avoid leaving mouth parts in the skin which can cause allergic reaction. Safe tick removal helps to prevent pathogen transmissions and should be performed with fine-nosed tweezers (to avoid compression of the tick's body). ${ }^{28}$

\section{Repellents to avoid tick infestations}

One of the best repellents proven to effectively stop ticks in their tracks is DEET that can be buy in the form of sprays, lotions or creams. Persons can directly spray N,N-Diethyl-meta-toluamide also called DEET, on clothing and body while avoiding eyes and mouth, and never spray this repellent on sunburns, rashes, or cuts. Also, DEET and permethrin pre-treated clothing and fabrics are also available in the market and can be worn on hikes or nature walks as most of treated fabrics remain effective for up to 600 washes. ${ }^{29}$

\section{Removing of ticks immediately}

Removing a tick that has attached itself to human's skin requires skills and tools to avoid getting bitten. Never remove an attached tick using of our bare hands. Always use tweezers or a tissue paper to pull the tick out completely. Make sure the tick comes out completely and does not leave behind its mouth parts in the skin. Another effective way of removing a tick is using cellophane tape. The ends of the tape can be wrapped around the tick covered area and pull out with the tick stuck to it. ${ }^{30}$

\section{Avoiding of tick bites using protective clothing}

The best way to avoid tick bites is to wear long sleeved shirts, full pants, socks and shoes. Ticks also can be seen easily on lighter colored 
clothes. It is also a good idea to tape open sleeves or loose ends so that ticks cannot crawl inside them. ${ }^{31}$

\section{Get treated immediately}

Some of tick-borne diseases can be effectively treated and cured using antibiotics provided to seek treatment in its early stages. Delaying of treatment can lead to permanent after-effects of the disease. Chronic conditions like joint and muscle aches have been reported in patients and they take years to improve. ${ }^{32}$

\section{Anti-tick vaccine}

Research in the post-genomic era is leading to the development of new control measures such as the recombinant vaccines. Tick infestation is rarely a one-species issue and therefore, anti-tick vaccines should aim at a more global protection against the main pathogen species of economical and epidemiological interest. The great rise of acaricide resistance, still asks for the implementation of an effective vaccine. The discovery of potential antigens against tick's bit and tick-pathogens proteins should result in improved production of vaccines, more advantageous in an overall approach to control tickborne diseases. ${ }^{33}$

\section{Conclusion}

This article concerns to the information on the methods used for tick and tick-borne parasites control and is mainly focused on the development of new technique to protect human. Soft ticks are distributed worldwide and global climatic changes, along with social factors, influence tick's habitats and their hosts. Of the estimated 117 species of ticks, 36 have been confirmed to carry or transmit one or more pathogens and 24 additional species are known to be pathogenic vectors in some countries. Furthermore, 38 species have been shown to carry multiple pathogens, indicating the major roles they play in the spread and transmission of these pathogens. Therefore, the number of pathogens and the vector potential of ticks may still be underestimated, because of the complex distributions and the great diversity of tick species in diverse ecological habitats. Tick-borne diseases are since long known, but often neglected or progressively being recognized due to their economic impact in livestock, but also due to their impact in human health, to which they have become a threat. Under these circumstances, tick vectors may enhance their potential to spread bacteria, viruses, protozoa and helminthes. Endemic zones for a specific tick-borne pathogen may serve as the origin for its epidemiological dissemination towards new environments and this dissemination probably would require the adaptation of both the pathogen and the new vector to each other. However, such knowledge might provide clues to the further identification of tickassociated pathogens, especially in epidemic areas with multiple tick species. Targeted prevention methods would then be more effective in controlling of tick-borne diseases. Vector and reservoir surveillance is an important component of such a strategy. Remove of ticks carefully with forceps by pulling them out steadily and firmly until the tick comes out is a key step. Subsequently there is no single ideal solution for the control of ticks, so, an integrated control approach is probably the most effective step. Much more work is required to better distinguish between ticks that carry potential pathogens and those that are competent to transmit pathogens to a host.

\section{Acknowledgments}

None.

\section{Conflicts of interest}

Author declares there are no conflicts of interest.

\section{Funding}

None.

\section{References}

1. Peter RJ, Van den Bossche P, Penzhorn BL, et al. Tick, fly, and mosquito control-lessons from the past, solutions for the future. Vet Parasitol. $2005 ; 132(3): 205-215$.

2. Sarwar M. Ticks (Arachnida: Acari) induced Paralysis in Humans and Control of Incidence in the Current Civilization. International Journal for Research in Social Science and Humanities Research. 2016;1(7):27-36.

3. Sarwar MH, Sarwar M. Medical Importance of Ticks Bite and Diseases Transmission by Means of It Affecting Humans. Biomedical and Health Informatics. 2016;1(2):44-51.

4. Guglielmone AA, Robbins RG, Apanaskevich DA, et al. The Argasidae, Ixodidae and Nuttalliellidae (Acari: Ixodida) of the world: A list of valid species names. Zootaxa. 2010;2528:1-28.

5. De la Fuente J, Estrada-Peña A, Venzal JM, et al. Overview: ticks as vectors of pathogens that cause disease in humans and animals. Front Biosci . 2008;1(13):6938-6946.

6. Wu XB, Na RH, Wei SS, et al. Distribution of tick-borne diseases in China. Parasit Vectors. 2013;6:119.

7. Manzano-Román R, Díaz-Martín V, De la Fuente J,et al. Soft Ticks as Pathogen Vectors: Distribution, Surveillance and Control. Parasitology. 2012. p.125-162

8. Mans JB, Gothe R, Neitz AWH. Tick toxins: perspectives on paralysis and other forms of toxicoses caused by ticks. In: Alan S Bowman \& Pat Nuttall (Eds.), Ticks: biology, disease and control, Cambridge University Press, Cambridge, UK. 2008. p.92-107.

9. Molyneux DH. Vectors. In: Francis EG Cox ( $2^{\text {nd }}$ edn $)$, Modern parasitology: a textbook of parasitology Wiley-Blackwell, USA. 1993. p.53-74.

10. Sonenshine DE. Biology of ticks. Oxford University Press, New York, USA. 1992. p.447.

11. Vial L. Biological and ecological characteristics of soft ticks (Ixodida: Argasidae) and their impact for predicting tick and associated disease distribution. Parasite. 2009;16 (3):191-202.

12. Schwan TG, Corwin, Brown SJ. Argas (argas) monolakensis, new species (Acari: Ixodoidea: Argasidae), a parasite of California gulls on islands in mono lake, California: description, biology, and life cycle. $J$ Med Entomol. 1992;29(1):78-97.

13. Sonenshine DE, Mather TN. Ecological dynamics of tick-borne zoonoses, Oxford, Oxford University Press, India. 1994.

14. Samish M, Ginsberg H, Glazer I. Anti tick biological control agents: assessment and future perspectives. In: Alan S Bowman \& Pat Nuttall (Eds.), Ticks: biology, disease and controlpp. Cambridge University Press, Cambridge, UK. 2008. p.47-469.

15. Hoskins JD. Ixodid and Argasid Ticks: Keys to their Identification. Veterinary Clinics of North America: Small Animal Practice. 1991;21 (1):185-197

16. Abbassy MM, Osman M, Marzouk AS. West Nile virus (Flaviviridae: Flavivirus) in experimentally infected Argas ticks (Acari: Argasidae). Am J Trop Med Hyg. 1993;48:726-737. 
17. Turell MJ, Mores CN, Lee JS, et al. Experimental transmission of Karshi and Langat (tick-borne encephalitis virus complex) viruses by Ornithodoros ticks (Acari: Argasidae). J Med Entomol . 2004;41(5):973-977.

18. Abbassy MM, Stein KJ, Osman M. New artificial feeding technique for experimental infection of Argas ticks (Acari: Argasidae). J Med Entomol . 1994;31:202-205

19. Hutcheson HJ, Gorham $\mathrm{CH}$, Machain-Williams C, et al. Experimental transmission of West Nile virus (Flaviviridae: Flavivirus) by Carios capensis ticks from North America. Vector-Borne Zoonotic Dis. 2005;5(3):293-295.

20. Reeves WK, Loftis AD, Sanders F, et al. Borrelia, Coxiella, and Rickettsia in Carios capensis (Acari: Argasidae) from a brown pelican (Pelecanus occidentalis) rookery in South Carolina, USA Exp Appl Acarol. 2006;39(3-4):321-329.

21. Gould EA, Solomon T. Pathogenic flaviviruses. Lancet 2008;371(6911):500-509.

22. Mumcuoglu KY, Banet-Noach C, Malkinson M, et al. Argasid ticks as possible vectors of West Nile virus in Israel. Vector Borne Zoonotic Dis. 2005;5(1):65-71.

23. Nava S, Lareschi M, Rebollo $\mathrm{C}$, et al. The ticks (Acari: Ixodida: Argasidae, Ixodidae) of Paraguay. Annals of Tropical Medicine and Parasitology. 2007;101 (3):255-270.

24. Lawrie CH, Uzcategui NY, Gould EA, et al. Ixodid and Argasid Tick Species and West Nile Virus. Emerg Infect Dis. 2004;10(4):653-657.

25. Sarwar M, Sarwar MH, Khan MA. Crimean Congo Hemorrhagic Fever and Its. Prevention in Humans through Tick Vectors Control. Biomed Rep. 2017;3(3):16-22.
26. Oleaga-Perez A, Perez-Sanchez R, Encinas-Grandes A. Distribution and biology of Ornithodoros erraticus in parts of Spain affected by African swine fever. Vet Rec. 1990;126(2):32-37.

27. Ostfeld RS, Price A, Hornbostel VL, et al. Controlling ticks and tickborne zoonoses with biological and chemical agents. Bioscience. 2006;5:383-394.

28. Diaz JH. A 60-Year Meta-Analysis of Tick Paralysis in the United States: A Predictable, Preventable, and Often Misdiagnosed Poisoning. J Med Toxicol . 2010;6(1):15-21.

29. Nicholson W, Allen KE, McQuiston JH, et al. The Increasing Recognition of Rickettsial Pathogens in Dogs and People. Trends in Parasitol. 2010;26(4):205-212.

30. George JE, Pound JM, Davey RB. Chemical control of ticks on cattle and the resistance of these parasites to acaricides. Parasitol. 2004;129 Suppl:353-366.

31. Graf JF, Gogolewski R, Leach-Bing N, et al. Tick control: an industry point of view. Parasitol. 2004;129 Suppl:S427-S442.

32. Rajput ZI, Hu S, Chen W, et al. Importance of ticks and their chemical and immunological control in livestock. J Zhejiang Univ Science B. 2006;7(11):912-921.

33. Domingos A, Antunes S, Borges L, et al. Approaches towards tick and tick-borne diseases control. Rev Soc Bras Med Trop. 2013;46(3):265269 